\title{
An integrated peach genome structural variation map uncovers genes associated with fruit traits
}

Jian Guo ${ }^{1,2 \dagger}$, Ke Cao ${ }^{1 \dagger}$, Cecilia Deng ${ }^{3}$, Yong Li ${ }^{1}$, Gengrui Zhu' ${ }^{1}$, Weichao Fang ${ }^{1}$, Changwen Chen ${ }^{1}$, Xinwei Wang ${ }^{1}$, Jinlong $\mathrm{Wu}^{1}$, Liping Guan ${ }^{1}$, Shan $\mathrm{Wu}^{4}$, Wenwu Guo ${ }^{2}$, Jia-Long $\mathrm{Yao}^{3^{*}}$, Zhangjun Fei ${ }^{4,5^{*}}$ and Lirong Wang ${ }^{1^{*}}$

\author{
*Correspondence: jia-long.yao@ \\ plantandfood.co.nz; zf25@cornell. \\ edu; wanglirong@caas.cn \\ †Jian Guo and Ke Cao contributed \\ equally to this work. \\ ${ }^{3}$ The New Zealand Institute for \\ Plant \& Food Research Limited \\ Private Bag 92169, Auckland 1142, \\ New Zealand \\ ${ }^{4}$ Boyce Thompson Institute for Plant \\ Research, Cornell University, Ithaca, \\ NY, USA \\ ${ }^{1}$ Zhengzhou Fruit Research Institute, \\ Chinese Academy of Agricultural \\ Sciences, Zhengzhou, China \\ Full list of author information is \\ available at the end of the article
}

\begin{abstract}
Background: Genome structural variations (SVs) have been associated with key traits in a wide range of agronomically important species; however, SV profiles of peach and their functional impacts remain largely unexplored.

Results: Here, we present an integrated map of 202,273 SVs from 336 peach genomes. A substantial number of SVs have been selected during peach domestication and improvement, which together affect 2268 genes. Genome-wide association studies of 26 agronomic traits using these SVs identify a number of candidate causal variants. A 9-bp insertion in Prupe.4G186800, which encodes a NAC transcription factor, is shown to be associated with early fruit maturity, and a 487-bp deletion in the promoter of PpMYB10.1 is associated with flesh color around the stone. In addition, a $1.67 \mathrm{Mb}$ inversion is highly associated with fruit shape, and a gene adjacent to the inversion breakpoint, PPOFP1, regulates flat shape formation.
\end{abstract}

Conclusions: The integrated peach SV map and the identified candidate genes and variants represent valuable resources for future genomic research and breeding in peach.

Keywords: SVs, GWAS, Peach, Fruit, Fruit shape

\section{Introduction}

Structural variations (SVs), comprising insertions/deletions (indels), duplications, inversions, and translocations, are widely present in genomes [1-4]. In humans, SVs provide an extensive source of genetic variation for the identification of genes involved in important biological processes [5]. In many plant species, SVs have been reported to regulate agronomic traits such as fruit shape in tomato (Solanum lycopersicum) [6], nematode resistance in soybean (Glycine max) [7], reproductive morphology in cucumber (Cucumis sativus) [8], asexual reproduction in citrus [9], and fruit texture in peach (Prunus persica) [10]. Recent rapid advances in next-generation sequencing technologies have facilitated genome-wide detection of SVs in large crop populations [11, 12].

(c) The Author(s). 2020 Open Access This article is licensed under a Creative Commons Attribution 40 International License which permits use, sharing, adaptation, distribution and reproduction in any medium or format, as long as you give appropriate credit to the original author(s) and the source, provide a link to the Creative Commons licence, and indicate if changes were made. The images or other third party material in this article are included in the article's Creative Commons licence, unless indicated otherwise in a credit line to the material. If material is not included in the article's Creative Commons licence and your intended use is not permitted by statutory regulation or exceeds the permitted use, you will need to obtain permission directly from the copyright holder. To view a copy of this licence, visit http://creativecommons.org/licenses/by/4.0/. The Creative Commons Public Domain Dedication waiver (http://creativecommons.org/publicdomain/zero/1.0/) applies to the data made available in this article, unless otherwise stated in a credit line to the data. 
Peach is the fourth largest deciduous fruit crop in the world (FAO, http://faostat.fao. org) and is regarded as a model plant for the Rosaceae family, thanks to its small genome and relatively short juvenile period [13]. Linkage analysis in peach has identified candidate genes for traits such as fruit flesh color [14], fruit hairiness [15], fruit flesh texture [10], double flower shape [16], and pendulous branches [17]; however, the genetic basis of these and other agronomic traits still remains largely unexplored. Genome-wide association studies (GWAS) represent an efficient method for mapping candidate genes and have been applied in peach to identify genome regions and/or genes associated with important traits, using genome-wide single nucleotide polymorphism (SNP) data [18-20]. However, identification of causal genes/variants is challenging in cases with large candidate regions. Furthermore, most GWAS analyses to date have been based on SNP data, while many phenotypes are associated with SVs. In peach, all reported genes that might be responsible for target traits have been associated with SVs [10, 15-17]. A pioneer study using SV data for GWAS in plants was reported in cucumber, where a large duplication controlling the reproductive morphology trait was detected [8]. Such an approach represents a more direct method for identifying candidate genes and casual variants, and the development of new bioinformatics methodologies, including more tools for analyzing SVs, has further enabled genome-wide SV mining.

Even though SVs are important sources of peach genetic diversity, their impact on genes and agronomic traits is still largely unknown. To date very few studies have focused on genome-wide detection of peach SVs [20], and subsequent investigations into the relationship between SVs and specific phenotypes using GWAS have not been reported. In this current study, 336 peach accessions were used to evaluate the SV landscape across the peach genome, resulting in an integrated SV map of more than 200,000 variants. GWAS of 26 agronomic traits were performed using the detected SVs in order to determine their significance and functional impacts.

\section{Results and discussion}

\section{A peach sequence-based SV map}

A collection of 336 peach accessions, originating from all over the world (Fig. 1a), were re-sequenced to an average depth of $24 \times$ using the Illumina Hiseq X Ten platform (Additional file 1: Table S1). In order to improve the SV detection accuracy, we used four different tools that show high performance for human SV detection [21, 22]: LUMPY [23], Manta [24], GRIDSS [25], and Delly [26]. To further reduce the falsepositive rate, SVs detected by LUMPY were filtered by setting a threshold for quality value $(>20)$ [21] and number of supporting reads (>5). Deletions smaller than $340 \mathrm{bp}$ lacking split read support were removed, and only variants detected by at least two tools were kept for the downstream analyses.

A final set of 202,273 SVs, ranging from $50 \mathrm{bp}$ to $10 \mathrm{Mb}$, were identified among the 336 peach genomes, including 121,527 indels, 10,728 duplications, 8336 inversions, and 61, 682 translocations (Additional file 1: Table S2). Based on these identified SVs, the peach accessions studied here could be divided into three major groups, ornamental cultivars and landraces as one group, the improved cultivar group, and the wild group (Fig. 1b, c, Additional file 2: Fig. S1). Phylogenetic relationship of the accessions inferred from these data was largely consistent with that reported in previous studies inferred using SNPs 


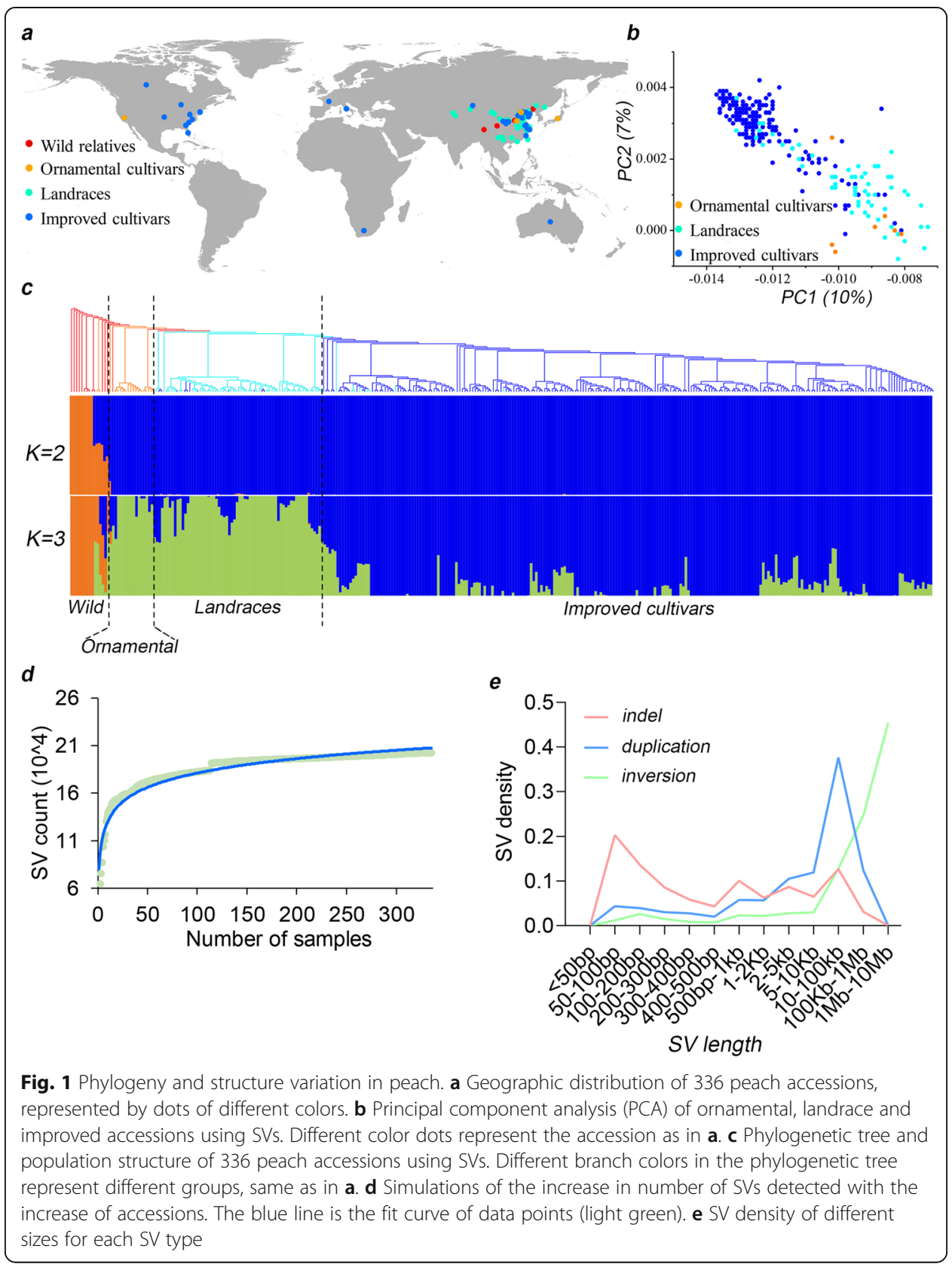

[18-20]. The total number of SVs from 150 randomly selected accessions was 193,575, accounting for $96 \%$ of the total SV number in all 336 accessions. Modeling of the SV size by iteratively randomly sampling accessions indicated that the SV number was relatively finite in the peach population (Fig. 1d), suggesting that our SV detection was comprehensive and nearly complete. Most of the detected SVs were present in a few accessions, and a total of 131,075 SVs had a minor allele frequency (MAF) $<0.01$ (Additional file 2: Fig. S2). Most indels and duplications ranged in size from $100 \mathrm{bp}$ to $10 \mathrm{~kb}$, while many inversions were $>10 \mathrm{~kb}$ (Fig. 1e, Additional file 1: Table S2).

Short length of reads generated using next-generation sequence (NGS) technologies and limitations of current computational algorithms can restrain the accuracy of SV detection [27, 28]. To assess the accuracy of the SV calling in this study, six of the 336 
accessions were sequenced using PacBio single molecule real-time sequencing (SMRT) technology [29]. A list of 150 SVs were randomly selected. A manual check by comparing these SVs with the PacBio long read mapping results using the IGV program [30] revealed an accuracy rate of $88 \%$ (Additional file 1: Table S3).

\section{Functional impact of peach SVs}

SVs are known to have a major influence on genomes and are often associated with specific traits [5]. We investigated the associations of the SVs identified here with gene, coding sequence (CDS), and promoter regions. A total of 26,361 out of 26,873 genes (98\%) were associated with at least one SV among the 336 peach genomes (Additional file 1: Table S4). These included 20,721 in CDSs, 3505 in introns, and 2135 in promoter regions. The 512 unaffected genes in the peach genome were mostly annotated as related to fundamental biological processes, such as chloroplast function (Additional file 2: Fig. S3, Additional file 1: Table S4), indicating genome regions regulating these processes have been fixed during peach evolution. This result indicated that SVs have a major role in gene regulation and morphological variation. This phenomenon has also been reported in other pan-genome studies [31,32], where genes that were not present in the reference genome sequence were identified due to the presence of SVs.

During peach domestication and improvement, many genetic loci have been selected, and a number of studies have characterized this phenomenon using SNP data [19, 20]. In this study, a total of 134 domestication and 97 improvement sweeps, covering 10.3\% $(22.9 \mathrm{Mb})$ and $8.7 \%(20.0 \mathrm{Mb})$ of the genome, respectively, were identified using SNPs, while a total of 210 domestication and 170 improvement sweeps, covering $13.6 \%$ (31.3 $\mathrm{Mb}$ ) and $12.1 \%(28.0 \mathrm{Mb}$ ), respectively, were identified using SVs (Additional file 2: Fig. S4, Additional file 1: Table S5-8). There were only $11.7 \mathrm{Mb}$ overlapping sweeps for domestication and $11.7 \mathrm{Mb}$ for improvement between those identified using SNPs and SVs (Additional file 1: Table S9-10). Therefore, to evaluate the impact of SVs on domestication and improvement processes, we selected specific SV sites based on their occurrence frequency and calculated and compared the occurrence frequencies for each SV in wild, landrace, and improved accession groups. Among all the detected SVs, 25,416 showed evidence of positive selection during domestication, with occurrence frequencies significantly higher in the landrace group than in the wild, while 29,780 showed evidence of negative selection (Fig. 2a, b, Additional file 1: Table S11). During the improvement process from landrace to modern cultivars, 4994 and 22,277 SVs were positively and negatively selected, respectively (Fig. 2c, d, Additional file 1: Table S11). The putative functional impact of these SVs was examined, and $<5 \%$ were found to be located in gene regions (Fig. 2e, f), affecting 2123 genes in the domestication process and 1207 genes in the improvement process. Gene ontology $(\mathrm{GO})$ enrichment analysis showed that genes involved in cellular response to stimulus and signaling were significantly overrepresented (Fig. 2g, h), which is in agreement with a similar study in tomato [31]. A total of 1093 genes showed evidence of positive selection and 1432 of negative selection during domestication, with the corresponding numbers being 275 and 1023, respectively, during the improvement process (Additional file 1: Table S11). In total, 2268 genes have been selected during peach evolution, with 1059 specifically during domestication, 145 specifically during improvement, and 1064 during both (Additional file 2: Fig. S5). 


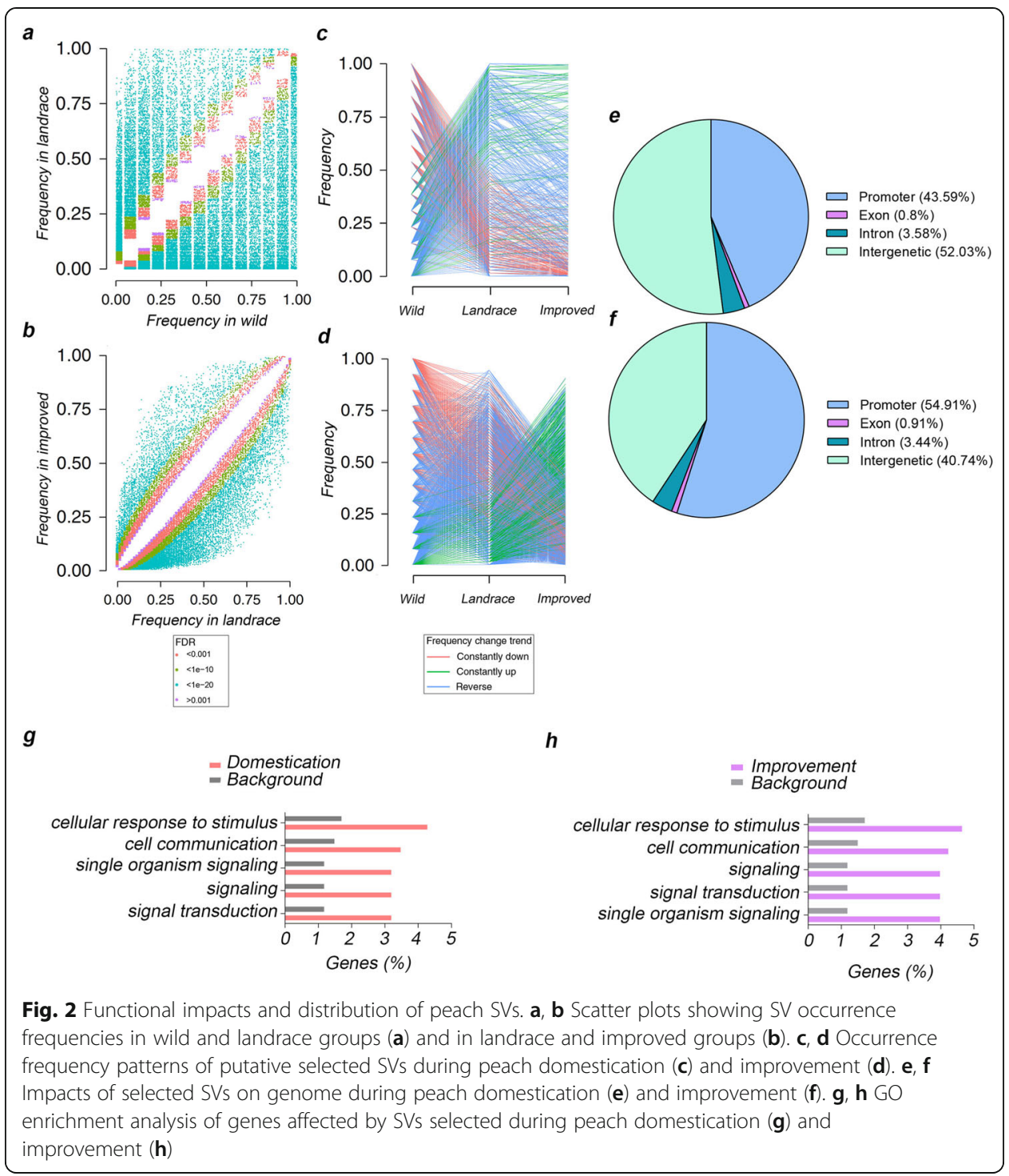

\section{Genome-wide association studies of $\mathbf{2 6}$ agronomic traits}

To further characterize functions of the SVs in peach, GWAS were performed for 26 key agronomic traits, including 18 traits for which GWAS has not been previously reported in peach. Performing GWAS with SNPs is an established methodology and one that takes advantage of the fact that SNPs provide a much higher density of polymorphisms than SVs and can be mapped to a relatively accurate genomic interval $[9,19$, 33-35]. However, only a few studies in plants to date have described GWAS using SVs $[8,32]$. There is considerable evidence from peach genetic and molecular studies that SVs can cause major phenotypic variance [10, 15-17], suggesting that SVs may represent a valuable source of variation for GWAS in peach. In order to characterize causal SVs, GWAS were performed separately using SNPs and SVs (including small variations $<50 \mathrm{bp}$ ) with MAF > 0.01. A total of 71,198 large SVs (> $50 \mathrm{bp)}$ ) combined with 109,067 small indels $(<50 \mathrm{bp})$ with MAP $>0.01$ were used for GWAS. The highly associated regions identified with SVs were largely consistent with those identified using SNPs and were also largely consistent using different models (Additional file 2: Fig. S6-31). In 
this study, GWAS provided reliable candidate variants that were used for gene identification in the context of these 26 agronomic traits (Additional file 2: Fig. S6-31, Additional file 1: Table S12).

\section{GWAS using SVs provide a powerful approach for identifying candidate genes}

We selected 8 qualitative traits that have been targeted in previous GWAS analyses using SNPs $[19,20]$ and performed GWAS for them using both SNPs and SVs. We found that the most significant association signals were almost in the same genome regions between the two datasets; however, GWAS using SV data link traits to large genome variations, which are more likely to alter gene functions. For the 11 qualitative traits analyzed in this study, candidate genes have been previously found for four of them, namely fruit hairiness [15], flesh color [14], double flower shape [16], and pendulous branches [17], and we also identified these candidate causal DNA variations using GWAS with the SV data (Additional file 2: Fig. S7, S8, S10, S12, Additional file 1: Table S12). A transposable element (TE) insertion in a MYB gene (Prupe.5G196100) was previously reported to be associated with the fruit hairiness trait, resulting in the peach and nectarine phenotypes [15], and this variation was also found to be the most significant signal in the GWAS with SV data (Additional file 2: Fig. S7). For the fruit flesh color trait, three variations in the CCD4 gene (Prupe.1G255500) on chromosome 1 have been implicated: a TE insertion, a simple sequence repeat (SSR), and a SNP [14]. Since multiple loci contribute to this trait, GWAS is unlikely to identify all three variants at the same time, and we found the most significant signal to be the SSR locus (Additional file 2: Fig. S8). When checking for variants located in this gene in 279 accessions with known flesh color phenotypes, we also found that these three variants were associated with the flesh color trait. Finally, a 1.3-kb deletion in Prupe.3G200700 was found to be related to the pendulous branch trait (Additional file 2: Fig. S12, Additional file 1: Table S12). However, since this locus was not the most significant signal in the GWAS analysis, and some accessions with a weeping phenotype did not contain this variation, indicating that other variants responsible for the pendulous branch trait might exist. Taking all these into account, we concluded that GWAS using SVs provides an efficient strategy to identify candidate genes and in some cases can outperform the traditional GWAS with SNP approach, in agreement with studies of rapeseed (Brassica napus) [32].

There is considerable evidence that SVs control many phenotypic traits [28, 36, 37], and GWAS represents a powerful tool for mapping genes in genetic and molecular biology studies [19, 34, 35, 38, 39]. Due to the reliable detection of SNPs, GWAS using SNPs has been widely used; however, there are only a few studies showing that a single SNP can change gene function and the phenotype. In peach, only one trait (flesh color, white/yellow) was reported to be controlled by a SNP, while the flesh color trait was also controlled by two other non-SNP variations [14]. All other reported traits in peach were regulated by SVs. In our study, GWAS were performed using SVs, resulting in a map that was similar to that from GWAS using SNPs, but GWAS with SVs here allowed a more accurate prediction of causal variants that might be responsible for traits.

\section{A 9-bp insertion in a gene coding region leads to early fruit maturity}

Fruit maturity date (MD) is a critical factor in fruit marketing, and previous studies using linkage analysis have placed the MD locus on linkage group 4 in peach [40, 41]. 
A possible candidate gene, Prupe.4G186800, encoding a NAC transcription factor, was reported to be responsible for the MD trait in peach, and a 9-bp insertion was identified as the possible causal variant [41]. Consistently, this insertion in the coding region of Prupe.4G186800 was also detected as the most significant signal in the GWAS analysis (Fig. 3a); however, Prupe.4G186800 and Prupe.4G186900 (annotated as encoding a protein with unknown function) were in the same linkage disequilibrium (LD) block (Fig. 3a). By genotyping this variant in the population, the accessions could be divided into three groups: early $\mathrm{MD}$, which contains a homozygous insertion (1/1); intermediate $\mathrm{MD}$, with a heterozygous genotype $(0 / 1)$; and late $\mathrm{MD}$, with no insertion, which is the same genotype as the reference (0/0) (Fig. 3b). To validate the functional role of Prupe.4G186800 in controlling MD and given the transgenic limitations in peach, the cultivar "Spring Snow" (SS) and its bud mutant "Jinlei" (JL), whose fruit matures 15 days earlier than "SS," and the "NJC83" cultivar and its bud mutation "Huihuang" (HH), whose fruit matures 10 days earlier, were subjected to comparative transcriptome analysis of fruit development. The differentially expressed genes (DEGs) in a 200-kb genome interval around the candidate region were analyzed, and Prupe.4G186800 was the only gene with much higher expression in both bud mutants (Fig. 3c, d). During fruit development, this gene showed much higher expression levels in early stages (Fig. 3c, d). In addition, this locus also provided the most significant signal in GWAS for the fruit development period trait (Additional file 2: Fig. S21). This finding suggested that fruit MD and the fruit growth period trait are correlated. In summary, we confirmed that MD located in linkage group 4 is a major MD locus and that Prupe.4G186800 is a candidate gene and the 9-bp insertion in Prupe.4G186800 is a candidate causal variant for early fruit maturity and fruit growth control.

\section{Gain of anthocyanins in flesh surrounding the fruit stone}

Anthocyanin accumulation is an important fruit attribute, and candidate genes for anthocyanin-related fruit traits have been reported in peach, including flesh color [42, 43] and fruit skin color [44]. The main candidate gene for fruit skin color is $P p M Y B 10.1$, while more than one gene, but including PpMYB10.1, have been associated with regulating flesh color $[42,43]$. The presence of one signal for fruit skin color and many for flesh color was also noted here (Additional file 2: Fig. S32a, b). However, the underlying mechanism determining flesh color around the stone is still unknown. From the GWAS analysis using SV data, a 487-bp deletion affecting the PpMYB10.1 promoter region was found to be associated with this trait (Fig. 4a, b), and the deletion genotype was highly correlated with the red flesh around the stone phenotype (Fig. 4c). To investigate the potential contribution of $P p M Y B 10.1$ to the color formation in the flesh surrounding the stone, its expression was analyzed and found to correlate with the occurrence of anthocyanins in the corresponding flesh region (Fig. 4d). It has been reported that the expression of PpMYB10.1 is regulated by the BL transcription factor [38]; however, the effect of the deletion on the promoter activity of $P p M Y B 10.1$ remains unknown. In a dual-luciferase reporter assay, we found that the deletion enhanced the promoter activity, consistent with a role of PpMYB10.1 in flesh color formation around the stone (Fig. 4e). In addition, the deletion in the PpMYB10.1 promoter was found to have been selected for during domestication, with a significantly higher occurrence frequency in landraces than in wild accessions (Additional file 1: Table S11). 


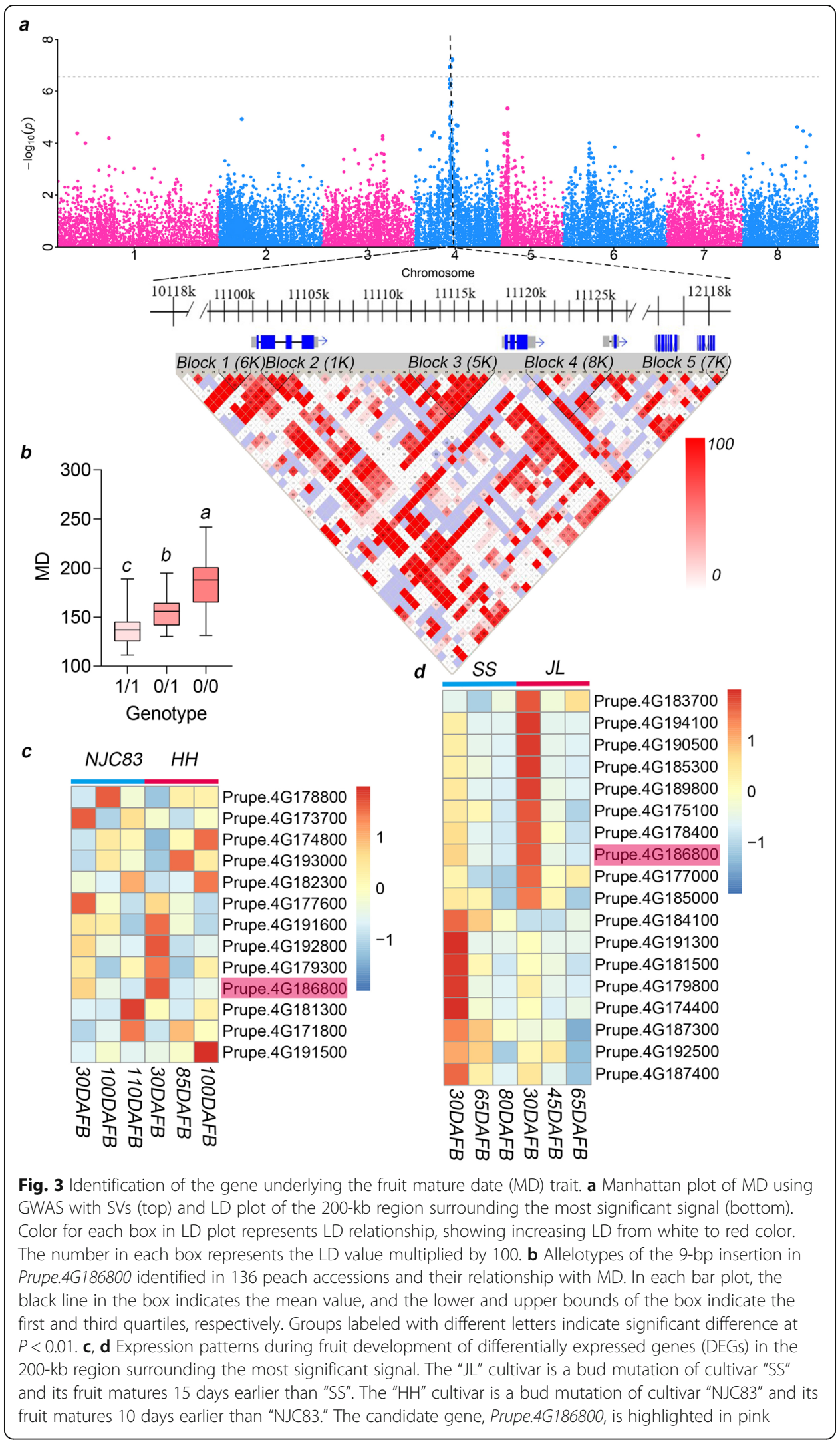




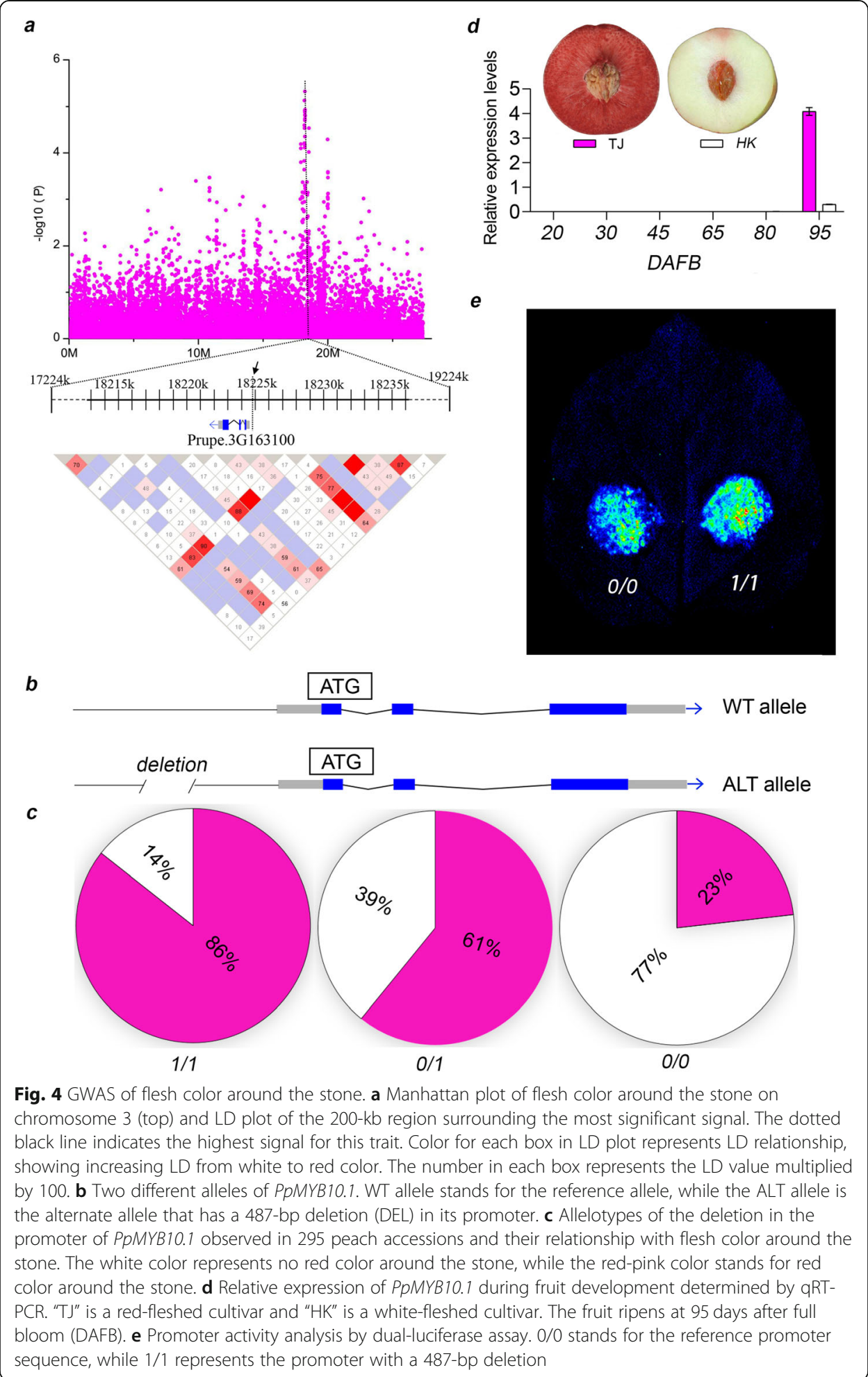

\section{A large inversion co-segregates with flat fruit shape}

Fruit shape is an important trait and determinant of consumer selection. Peach fruit have two typical shapes, flat and round, which are controlled by a single dominant gene, $S$ [45]. Genetic markers closely linked to the flat fruit trait have been identified 
and are useful for marker-assisted breeding; however, the specific mechanisms controlling flat shape formation in peach are still unknown. In this study, GWAS was performed for fruit shape using SV data and a $1.67-\mathrm{Mb}$ inversion was identified (Fig. 5a) that cosegregated with the flat fruit shape. All flat peach accessions that could produce mature fruits were heterozygous at this inversion locus, while fruit of the homozygous genotype aborted during early development. In this study, 34 of 35 flat peach accessions were heterozygous and one was homozygous, while all the 301 round peach accessions were homozygous, with the same genotype as the reference at this locus. To verify the accuracy of this inversion call, PacBio long reads spanning the breakpoints were identified, and meantime, a draft genome was assembled from PacBio long reads and contigs that spanned the inversion interval were identified from the assembly as well. The presence of the inversion was further confirmed by examining the alignments of raw reads (Additional file 2: Fig. S33a) and assembled contigs (Additional file 2: Fig. S33b), respectively, to the peach reference genome. We also validated this inversion in cultivars and an F1 population (a cross of "Okubo" $\times$ "You Pan Tao 1-3") using PCR-based method (Additional file 2: Fig. S34).

This inversion covers the SNP associated with flat shape reported in a previous study [19] and is approximately $500 \mathrm{~kb}$ away from the 10-kb deletion reported to co-segregate with the flat shape [46] (Additional file 2: Fig. S35). Based on its location, we deduced that the inversion might affect four genes, Prupe.6G290900, Prupe.6G291000, Prupe.6G323600, and Prupe.6G323700, which were located around the two breakpoints (Additional file 2: Fig. S35). To determine the best candidate gene, we performed quantitative real time-PCR (qRT-PCR) to measure the relative expression patterns of the four genes during fruit development in "JH" (round peach), "HY" (round peach), "ZPT10" (normal flat peach), "ZH" (normal flat peach), and "XJ2" (aborting flat peach). We found that the expression patterns of two of the genes, Prupe.6G290900 and Prupe.6G323700, were related to the allelic genotypes of flat and round peach (Fig. 5b, Additional file 2: Fig. S36), indicating that they might be responsible for flat fruit shape formation. Given that the flat shape gene is dominant, the candidate gene with higher expression levels in flat peaches was more likely to be the gene responsible for the flat phenotype, as was the case for Prupe.6G290900. Notably, Prupe.6G290900 was annotated as encoding an ovate family protein (OFP), a member of which is the key gene controlling fruit shape in tomato [47], and we named this gene PpOFP1. Phylogenetic analysis of OFP genes in peach and tomato showed a close relationship between PpOFP1 and SLOFP20, a gene known to control tomato fruit shape (Additional file 2: Fig. S37).

Stable transformation of peach is not yet technically possible and so we could not confirm the function of PpOFP1 by generating transgenic peach lines. As an alternative approach, we expressed PpOFP1 in the Micro-Tom tomato genotype. Ten transgenic lines with different levels of $P p O F P 1$ expression were generated (Fig. 5c). Three transgenic lines, OE3, OE4, and OE6, expressed $P p O F P 1$ to a medium to high levels and exhibited developmental aberrations. They were shorter than the WT control plants and most importantly produced flat tomato fruit (Fig. $5 \mathrm{~d}$, e). These lines also showed abnormal floral organs such as stamen and stigma (Fig. 5e, f). The stigma phenotype was similar to that of the flat peach stigma, which is shorter and thicker than those of round peach (Additional file 2: Fig. S38). According to paraffin section result, the cell number along the vertical axis in transgenic flat tomato fruit was lower than that in round tomato fruit (Fig. 5f,g), consistent with the development of flat peach [48]. 


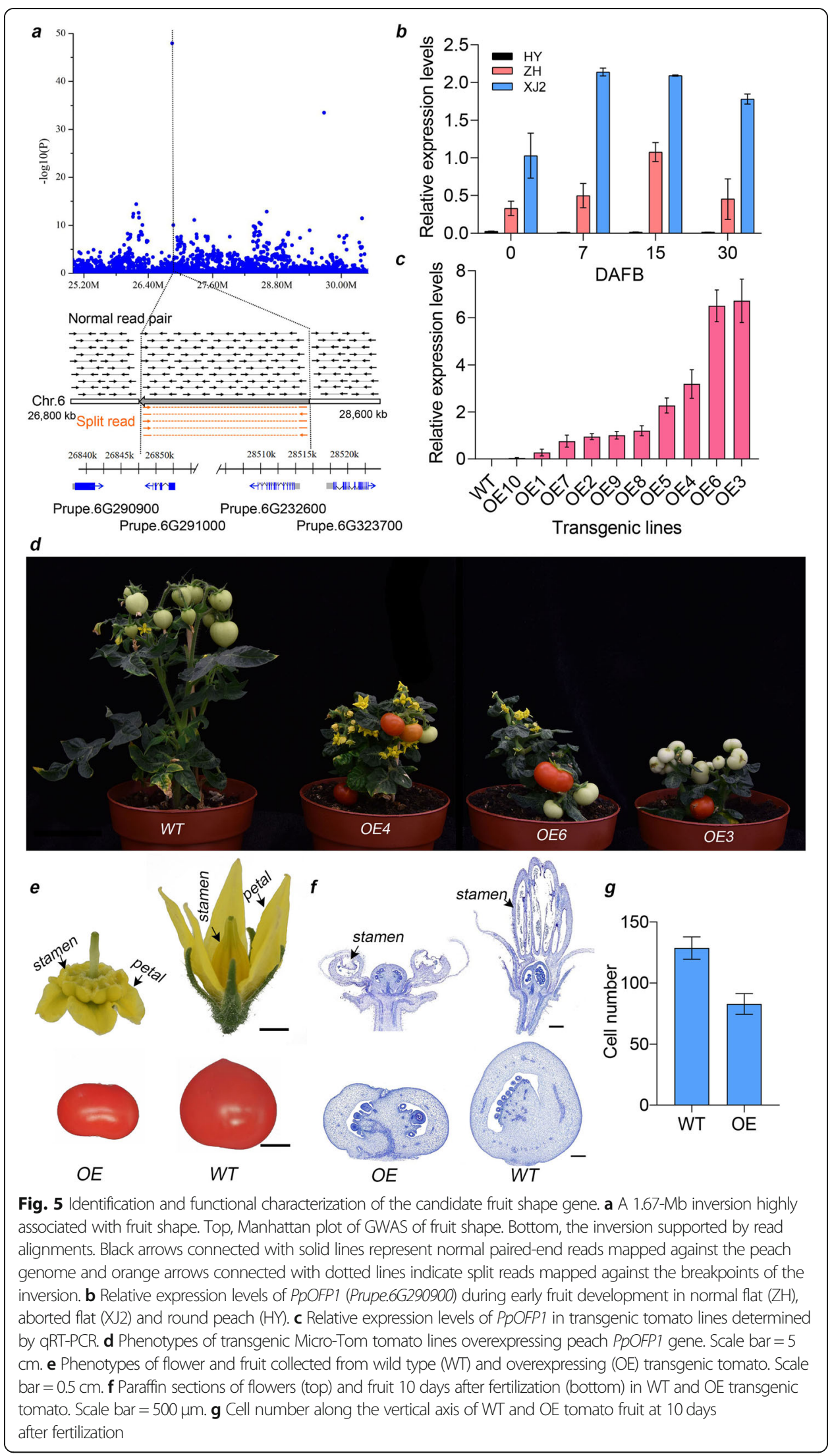




\section{Conclusions}

In this study, we provided an integrated map of genome SVs by re-sequencing 336 peach accessions, collectively originating from all over the world. We analyzed the putative effects of the SVs on the genome during peach domestication and improvement and found that almost all genes in peach were affected by SVs and that the very few unaffected genes were almost all involved in core biological processes. A GWAS approach using SVs was found to be more efficient than GWAS using SNPs in identifying candidate genes and causal variants, and based on the SV dataset we generated here, we performed GWAS for 26 peach agronomic traits. This suggested candidate genes responsible for the fruit related traits, such as flesh color around the stone, fruit MD, and fruit shape. The function of PpMYB10.1 was observed to include control of flesh color around the stone, while the candidate gene for fruit MD was confirmed to be Prupe.4G186800, as previously reported. The fruit shape candidate gene, $P p O F P 1$, was validated in transgenic tomato, and its heterologous expression in tomato leads to a flat fruit. The integrated SV map provides a valuable resource for future genomic research in peach and other plant species. In addition, the significant association signals identified for the 26 agronomic traits provide valuable candidates for the genetic improvement of peach and will be beneficial to the peach industry.

\section{Materials and methods}

\section{Plant materials and genome resequencing}

In this study, 336 peach accessions were sampled from National Peach Germplasm Repository of China (NPGRC, Zhengzhou), including 13 wild, 20 ornamental, 70 landrace, and 233 improved accessions (Additional file 1: Table S1). In these samples, 104 were reported in our previous study [20,49]; however, the sequencing depth of these accessions was $\sim 5 \times$, which would not be suitable for SV detection [23]. We therefore performed further genome resequencing of these accessions to an average depth of $\sim 20 \times$. The genomic DNA was extracted from young leaves as previously described [50]. DNA libraries were constructed with an insert size of $\sim 350 \mathrm{bp}$ and sequenced on a Hiseq X Ten platform (Illumina) which generated paired-end reads of $150 \mathrm{bp}$ in length. For each accession, no less than $5 \mathrm{~Gb}$ of sequencing data was generated to ensure the reliability of SV detection.

\section{Phenotyping}

Twenty-six agronomic traits were targeted in this study, including 11 qualitative and 15 quantitative traits. The 11 qualitative traits, including fruit shape, fruit hairiness, flesh color (white/yellow), flesh color around the stone, flower shape (double/single; showy/ non-showy), pendulous branch, pollen sterility, hypanthium color (white/yellow), anther color, and kernel taste, were measured in two successive years according to previous reported evaluation criteria [51] from 2011 to 2012. The 15 quantitative traits, including internode length, flower/leaf bud ratio, relative height between pistil and stigma, suture depth, fruit development period, development period, leaf length, leaf width, bloom date, full bloom date, bloom ending date, leaf expanding date, fruit maturity date, deciduous date, and deciduous ending date, were recorded in 1 to 3 years as shown in Additional file 1: Table S1. 


\section{SV detection and genotyping}

Raw Illumina reads were processed to remove adapter and low-quality sequences. The paired-end reads were mapped to the reference peach "Lovell" genome [52] (release version 2.0_a2.1) using BWA [53] (version 0.7.15) with the following parameters: "bwa mem -M -R." Four tools were selected for SV detection: LUMPY [23] (version 0.2.13), Manta [24] (version 1.6.0), GRIDSS [25] (version 2.5.2), and Delly [26] (version 0.7.8). LUMPY was used to detect SVs, with the exception of insertions, with the following parameters: "lumpyexpress -P -B -S -D." To further reduce the number of false SVs, we filtered deletions that were $<340 \mathrm{bp}$ and had no split read support. The SV results were then genotyped in the population using SVTyper [54] (version 0.0.4). For the other three tools, SVs were detected and genotyped using the default parameters, as these tools can be used to both detect SV and perform genotyping. To improve the accuracy, the results were filtered using SURVIVOR [55] (version 1.0.6) by keeping the variants that were detected by at least two tools, with the following parameters: "SURVIVOR merge name 10002110 50." SURVIVOR defines and merges SVs according to the distance between breakpoints, SV type and SV strands. In this study, we set the maximum distance between breakpoints to $1000 \mathrm{bp}$. Transposons were identified with panISa [56] and genotyped according to the number of clipped reads and sequencing depth at the variants position using an in-house script.

\section{Small variants calling}

SNP and small indel calling were performed using the GATK Best-Practices pipeline (https://software.broadinstitute.org/gatk/best-practices/). The detection was performed using the GATK HaplotypeCaller, and genotyping was done with GenotypeGVCFs [57], and the separation of SNPs and indels was performed using the GATK selectTypeToInclude option. After separation, the SNP call set was filtered by applying the following parameters in GATK VariantFiltration to ensure accuracy: "QUAL $<40$, QD $<2.0, F S>60.0$, MQ <40.0, MQRankSum <-12.5, ReadPosRankSum <-8.0." The indel ( $<50 \mathrm{bp}$ ) set was filtered with parameters: "QD <2.0, FS >200.0, ReadPosRankSum <-20.0."

\section{Quality evaluation of the detected peach SV set}

To evaluate the accuracy of the detected SVs, we randomly selected 150 variants using the Linux command "shuf -n." In addition to Illumina short reads, single-molecule real-time sequencing (SMRT) was used to generate PacBio long reads for six accessions: "Xinjiang Pan Tao \#2," "Hakuho," “Tianjin Shui Mi," "Ying Ge Tao," "Okubo," and "Tian Ren Tao." The PacBio reads were mapped to the reference peach genome using NGMLR [58] (version 0.2.7). Based on the SV type, genome position and SV size, we manually checked the short-read mapping results and the PacBio long-read mapping results using IGV [30] to evaluate the accuracy of SVs.

\section{Phylogeny, population structure, and selective sweep analyses}

A maximum-likelihood phylogenetic tree was constructed based on the binary SV data using IQ-TREE [59] with 1000 bootstraps. Using the same binary SV data, population structure was investigated using ADMIXTURE [60], and principal component analysis (PCA) was performed using the smartpca program of the EIGENSFOT [61] software 
(version: 6.0.1) with default settings. Nucleotide diversity $(\pi)$ was calculated for each group with VCFtools [62] (version 0.1.12) using a window size of $100 \mathrm{~kb}$ and a step size of $10 \mathrm{~kb}$. The selective sweeps during domestication and improvement were identified based on reduction of diversity (ROD). The $\pi_{\text {wild }} / \pi_{\text {landrace }}$ and $\pi_{\text {landrace }} / \pi_{\text {improved }}$ values were calculated and the top $5 \%$ windows with the highest ROD values were identified as selective sweeps during domestication and improvement processes, respectively.

\section{Functional impact of SVs and SV selection during peach evolution}

According to the genome annotation, we assessed the putative SV impacts on gene, CDS, and promoter regions according to SV locations and sizes. We set an interval of $2 \mathrm{~kb}$ upstream of the gene as the promoter region. To evaluate the SV significance in peach evolution, the occurrence frequencies of each SV were calculated in wild, landrace and improved groups. SVs with higher or lower occurrence frequencies in landrace compared to wild, and improved compared to landrace, were designated as showing positive or negative selection, respectively, during domestication or improvement. The significance of the difference of the frequencies for each SV between the two compared groups (wild versus landrace for domestication and landrace versus improved for improvement) was determined using the Fisher exact test with the R package "pdrtool." The resulting raw $P$ values of all SVs in each of the two comparisons were then corrected based on a false discovery rate (FDR). SVs with significantly different frequencies (FDR $<0.001$ and fold change $>2$ ) were identified as those under selection. These SVs were then analyzed to identify the associated genes, and GO enrichment analysis was performed for these genes using the AgriGO [63] with a cutoff of FDR $<0.05$.

\section{GWAS for 26 agronomic traits}

To identify candidate genes responsible for the various agronomic traits, GWAS were performed for 26 agronomic traits. We aimed to identify variants associated with these traits by performing GWAS with the SV dataset and the SNP dataset, separately. To improve the accuracy of the GWAS results, we filtered the SV and SNP datasets by removing those with minor allele frequency $(\mathrm{MAF})<0.01$. GWAS were performed using MLM, CMLM, and FarmCPU models implemented in GAPIT [64] (version 3.0), which also integrates PCA and kinship analyses. The significance cutoff was defined as the Bonferroni test threshold, which was set as $0.05 /$ (total number of SVs) and $0.05 /$ (total number of SNPs), which corresponded to $-\log 10(\mathrm{P})=6.56$ for SVs and 7.82 for SNPs.

\section{Experimental validation of the inversion variation in the $S$ locus}

An inversion of $1.67 \mathrm{Mb}$ was found to co-segregate with the flat shape in peach. To validate this inversion, first, PacBio SMRT library was constructed and sequenced for the peach accession, Xinjiang Pan Tao \#2, which was homozygous at the $S$ locus. The resulting PacBio long reads were then mapped against the peach reference genome using NGMLR [58] and reads covering the inversion were identified according to the inversion location. Second, the PacBio reads were assembled de novo using Canu [65] (version 1.8) to obtain long contigs that might cover the inversion locus. After assembly, the collinearity between the assembled Xinjiang Pan Tao \#2 and the reference genomes was plotted using MUMmer [66] (version 3.9.4), and based on the dotplot around the $S$ locus, 
the inversion event was evaluated. Finally, we confirmed the inversion by PCR using primers (Additional file 1: Table S13) adjacent to the inversion breakpoints.

\section{RNA extraction and real-time PCR analysis}

To investigate the fruit maturity and flesh color around the stone traits, fruit samples were collected at five stages from "Hakuho" (HK; white flesh) and "Tianjin Shui Mi" (TJ; red flesh): 20, 40, 60, 80, and 90 DAFB (days after full bloom). For the fruit shape trait, fruit samples were collected at 16 stages from pre-bloom bud to fruit maturation period from "Zhong Tao Hong Yu" (HY; round peach) and "Zhong Pan Tao \#10" (ZPT10; normal flat peach). These samples were used to analyze candidate gene expression patterns. Samples from another two accessions, "Zao Huang Pao Tao" (ZH; normal flat peach) and "Xinjiang Pan Tao \#2" (XJ2; aborted flat peach), were collected at $0,7,15$, and $30 \mathrm{DAFB}$, to further validate the candidate genes. Total RNA was extracted from these fruit samples using an RNA-extracting kit (Hua Yue Yang, China). To check the relative expression levels of $P P O F P 1$ in transgenic tomato lines, RNA was extracted from young leaves using the same method. First- and second-strand complementary DNA (cDNA) samples were synthesized using a cDNA Synthesis System kit (TOYOBO, Japan) following the manufacturer's protocol. The qRT-PCR was performed using a LightCycler 480 (Roche) with the following cycle conditions: $95^{\circ} \mathrm{C}$ for $5 \mathrm{~min}$, followed by 45 cycles at $95^{\circ} \mathrm{C}$ for $10 \mathrm{~s}, 60^{\circ} \mathrm{C}$ for $10 \mathrm{~s}$ and $72{ }^{\circ} \mathrm{C}$ for $20 \mathrm{~s}$. The housekeeping gene RP-II (RNA polymerase II) was used as an internal control for peach [19]. The relative expression level was calculated using the $2^{-\Delta \Delta \mathrm{Ct}}$ method [67].

\section{Overexpression of the candidate gene in tomato}

The full-length coding region of $P p O F P 1$ was amplified from fruit cDNA sample of "XJ2" (aborting flat peach with homozygous genotype) by PCR using a high fidelity DNA polymerase (KOD-201, TOYOBO, Japan). The products were cloned into the $p B I 121$ vector downstream of the cauliflower mosaic virus (CaMV) $35 \mathrm{~S}$ promoter using a one-step construction kit (C112, Vazyme, China). The constructs were then transformed into MicroTom tomato using Agrobacterium tumefaciens GV3101 following protocol described in Sun et al. [68]. After transformation, transgenic lines were obtained and their fruit shapes were analyzed. Primers used in this experiment are listed in Additional file 1: Table S13.

\section{Microscopic analysis of transgenic tomato fruit and flowers}

Flower and fruit samples of transgenic tomato lines overexpressing PpOFP1 were collected at 0 and 10 DAFB and fixed in FAA immediately for 2 days. The samples were then removed from FAA, rinsed thoroughly in deionized water, and then dehydrated using an ethanol gradient, cleared using xylene, and embedded in wax. The embedded samples were then sectioned (10 $\mu \mathrm{m}$ section thickness), and the sections were rehydrated and stained using aqueous toluidine blue ( $\mathrm{pH}$ 7.0) [69]. Images were captured with a light microscope (Olympus) fitted with a camera (DP71, Olympus). To determine the cell number along the vertical axis, the cells were counted one by one manually under a light microscope. 


\section{Dual-luciferase reporter assay}

To determine whether the deletion in the promoter region of PpMYB10.1 affects the promoter activity, dual-luciferase reporter assays were carried out. Different promoter regions of PpMYB10.1 were cloned into pGreen-II-0800-LUC vector using the one-step construction kit as described above (C112, Vazyme, China). The promoters of PpMYB10.1 with or without the deletion were cloned into $p$ Green-II-0800-LUC to check the influence on gene expression. The constructs were transformed into GV3101 and transient expression assays were performed using tobacco leaves. The relative luciferase activities were detected and photos taken by a Tanon-5200Multi machine (Biotanon, China).

\section{Supplementary information}

Supplementary information accompanies this paper at https://doi.org/10.1186/s13059-020-02169-y.

Additional file 1. Supplementary tables S1-S13.

Additional file 2. Supplementary figures S1-S38.

Additional file 3. Review history.

\section{Acknowledgements}

This study was supported by the grants from the National Natural Science Foundation of China (31972392), the Agricultural Science and Technology Innovation Program (CAAS-ASTIP-2020-ZFRI-01), National Horticulture Germplasm Resources Center, and the US National Science Foundation (IOS-1855585 and IOS-1339287).

Peer review information

Kevin Pang was the primary editor on this article and managed its editorial process and peer review in collaboration with the rest of the editorial team.

\section{Review history}

The review history is available as Additional file 3.

\section{Authors' contributions}

L.W., Z.F., J-L.Y., and K.C. designed and managed the project. J.G., Y.L., G.Z., W.F., C.C., and X.W. collected materials and performed phenotyping. J.G., J.W., and L.G. prepared and purified DNA samples. J.G. and C.D. performed data analyses. J.G. wrote the paper. L.W., Z.F., J-L.Y., K.C., C.D., S.W., and W.G. revised the paper. All authors read and approved the final manuscript.

\section{Availability of data and materials \\ The sequence data have been deposited in NCBI Sequence Read Archive (SRA) under accession PRJNA630113 [70]. The SNPS and SVs in Variant Call Format (VCF) have been deposited in Zenodo [71]. The scripts used in this study were available in GitHub [72].}

\section{Ethics approval and consent to participate}

Not applicable.

\section{Consent for publication}

Not applicable.

\section{Competing interests}

The authors declare that they have no competing interests.

\section{Author details}

${ }^{1}$ Zhengzhou Fruit Research Institute, Chinese Academy of Agricultural Sciences, Zhengzhou, China. ${ }^{2}$ College of Horticulture \& Forestry Sciences, Huazhong Agricultural University, Wuhan, China. ${ }^{3}$ The New Zealand Institute for Plant \& Food Research Limited, Private Bag 92169, Auckland 1142, New Zealand. ${ }^{4}$ Boyce Thompson Institute for Plant Research, Cornell University, Ithaca, NY, USA. ${ }^{5}$ US Department of Agriculture-Agricultural Research Service, Robert W. Holley Center for Agriculture and Health, Ithaca, NY, USA.

Received: 20 March 2020 Accepted: 23 September 2020

Published online: 06 October 2020

\section{References}

1. Alkan C, Coe BP, Eichler EE. Genome structural variation discovery and genotyping. Nat Rev Genet. 2011;12:363-76.

2. lafrate AJ, Feuk L, Rivera MN, Listewnik ML, Donahoe PK, Qi Y, Scherer SW, Lee C. Detection of large-scale variation in the human genome. Nat Genet. 2004;36:949-51.

3. Tuzun E, Sharp AJ, Bailey JA, Kaul R, Morrison VA, Pertz LM, Haugen E, Hayden H, Albertson D, Pinkel D, et al. Fine-scale structural variation of the human genome. Nat Genet. 2005;37:727-32. 
4. Korbel JO, Urban AE, Affourtit JP, Godwin B, Grubert F, Simons JF, Kim PM, Palejev D, Carriero NJ, Du L, et al. Paired-end mapping reveals extensive structural variation in the human genome. Science. 2007;318(5849):420-6.

5. Sudmant PH, Rausch T, Gardner EJ, Handsaker RE, Abyzov A, Huddleston J, Zhang Y, Ye K, Jun G, Fritz MH, et al. An integrated map of structural variation in 2,504 human genomes. Nature. 2015;526:75-81.

6. Xiao H, Jiang N, Schaffner E, Stockinger EJ, Van Der Knaap E. A retrotransposon-mediated gene duplication underlies morphological variation of tomato fruit. Science. 2008;319(5869):1527-30.

7. Cook DE, Lee TG, Guo X, Melito S, Wang K, Bayless AM, Wang J, Hughes TJ, Willis DK, Clemente TE, et al. Copy number variation of multiple genes at Rhg1 mediates nematode resistance in soybean. Science. 2012;338(6111):1206-9.

8. Zhang Z, Mao L, Chen H, Bu F, Li G, Sun J, Li S, Sun H, Jiao C, Blakely R, et al. Genome-wide mapping of structural variations reveals a copy number variant that determines reproductive morphology in cucumber. Plant Cell. 2015;27: $1595-604$.

9. Wang X, Xu Y, Zhang S, Cao L, Huang Y, Cheng J, Wu G, Tian S, Chen C, Liu Y, et al. Genomic analyses of primitive, wild and cultivated citrus provide insights into asexual reproduction. Nat Genet. 2017;49:765-72.

10. Gu C, Wang L, Wang W, Zhou H, Ma B, Zheng H, Fang T, Ogutu C, Vimolmangkang S, Han Y. Copy number variation of a gene cluster encoding endopolygalacturonase mediates flesh texture and stone adhesion in peach. J Exp Bot. 2016;67:1993-2005.

11. Zhou Y, Minio A, Massonnet M, Solares E, LV Y, Beridze T, Cantu D, Gaut BS. The population genetics of structural variants in grapevine domestication. Nat Plants. 2019;5:965-79.

12. Yang N, Liu J, Gao Q, Gui S, Chen L, Yang L, Huang J, Deng T, Luo J, He L, et al. Genome assembly of a tropical maize inbred line provides insights into structural variation and crop improvement. Nat Genet. 2019:51:1052-9.

13. Shulaev V, Korban SS, Sosinski B, Abbott AG, Aldwinckle HS, Folta KM, lezzoni A, Main D, Arus P, Dandekar AM, et al. Multiple models for Rosaceae genomics. Plant Physiol. 2008;147:985-1003.

14. Adami M, De Franceschi P, Brandi F, Liverani A, Giovannini D, Rosati C, Dondini L, Tartarini S. Identifying a carotenoid cleavage Dioxygenase (ccd4) gene controlling yellow/white fruit flesh color of peach. Plant Mol Biol Report. 2013;31:1166-75.

15. Vendramin E, Pea G, Dondini L, Pacheco I, Dettori MT, Gazza L, Scalabrin S, Strozzi F, Tartarini S, Bassi D, et al. A unique mutation in a MYB gene cosegregates with the nectarine phenotype in peach. PLoS One. 2014;9:e90574.

16. Gattolin S, Cirilli M, Pacheco I, Ciacciulli A, Da Silva LC, Mauroux JB, Lambert P, Cammarata E, Bassi D, Pascal T, Rossini L. Deletion of the miR172 target site in a TOE-type gene is a strong candidate variant for dominant double-flower trait in Rosaceae. Plant J. 2018;96:358-71.

17. Hollender CA, Pascal T, Tabb A, Hadiarto T, Srinivasan C, Wang W, Liu Z, Scorza R, Dardick C. Loss of a highly conserved sterile alpha motif domain gene (WEEP) results in pendulous branch growth in peach trees. Proc Natl Acad Sci U S A. 2018;115:E4690-9.

18. Cao K, Zheng Z, Wang L, Liu X, Zhu G, Fang W, Cheng S, Zeng P, Chen C, Wang X, et al. Comparative population genomics reveals the domestication history of the peach, Prunus persica, and human influences on perennial fruit crops. Genome Biol. 2014;15(7):415.

19. Cao K, Zhou Z, Wang Q, Guo J, Zhao P, Zhu G, Fang W, Chen C, Wang X, Wang X, et al. Genome-wide association study of 12 agronomic traits in peach. Nat Commun. 2016;7:13246.

20. Li Y, Cao K, Zhu G, Fang W, Chen C, Wang X, Zhao P, Guo J, Ding T, Guan L, et al. Genomic analyses of an extensive collection of wild and cultivated accessions provide new insights into peach breeding history. Genome Biol. 2019;20:36.

21. Chiang C, Scott AJ, Davis JR, Tsang EK, Li X, Kim Y, Hadzic T, Damani FN, Ganel L, Consortium GT, et al. The impact of structural variation on human gene expression. Nat Genet. 2017:49:692-9.

22. Collins RL, Brand H, Redin CE, Hanscom C, Antolik C, Stone MR, Glessner JT, Mason T, Pregno G, Dorrani N, et al. Defining the diverse spectrum of inversions, complex structural variation, and chromothripsis in the morbid human genome. Genome Biol. 2017;18:36.

23. Layer RM, Chiang C, Quinlan AR, Hall IM. LUMPY: a probabilistic framework for structural variant discovery. Genome Biol. 2014;15(6):R84.

24. Chen X, Schulz-Trieglaff O, Shaw R, Barnes B, Schlesinger F, Kallberg M, Cox AJ, Kruglyak S, Saunders CT. Manta: rapid detection of structural variants and indels for germline and cancer sequencing applications. Bioinformatics. 2016;32: $1220-2$.

25. Cameron DL, Schroder J, Penington JS, Do H, Molania R, Dobrovic A, Speed TP, Papenfuss AT. GRIDSS: sensitive and specific genomic rearrangement detection using positional de Bruijn graph assembly. Genome Res. 2017;27: 2050-60.

26. Rausch T, Zichner T, Schlattl A, Stutz AM, Benes V, Korbel JO. DELLY: structural variant discovery by integrated pairedend and split-read analysis. Bioinformatics. 2012;28:1333-9.

27. Handsaker RE, Korn JM, Nemesh J, McCarroll SA. Discovery and genotyping of genome structural polymorphism by sequencing on a population scale. Nat Genet. 2011;43:269-76.

28. Mills RE, Walter K, Stewart C, Handsaker RE, Chen K, Alkan C, Abyzov A, Yoon SC, Ye K, Cheetham RK, et al. Mapping copy number variation by population-scale genome sequencing. Nature. 2011;470:59-65.

29. Rhoads A, Au KF. PacBio sequencing and its applications. Genomics Proteomics Bioinformatics. 2015;13:278-89.

30. Robinson JT, Thorvaldsdóttir H, Winckler W, Guttman M, Lander ES, Getz G, Mesirov JP. Integrative genomics viewer. Nat Biotechnol. 2011;29(1):24-6

31. Gao L, Gonda I, Sun H, Ma Q, Bao K, Tieman DM, Burzynski-Chang EA, Fish TL, Stromberg KA, Sacks GL, et al. The tomato pan-genome uncovers new genes and a rare allele regulating fruit flavor. Nat Genet. 2019;51:1044-51.

32. Song J-M, Guan Z, Hu J, Guo C, Yang Z, Wang S, Liu D, Wang B, Lu S, Zhou R, et al. Eight high-quality genomes reveal pan-genome architecture and ecotype differentiation of Brassica napus. Nature Plants. 2020;6:34-45.

33. Ganal MW, Altmann T, Roder MS. SNP identification in crop plants. Curr Opin Plant Biol. 2009;12:211-7.

34. Huang X, Wei X, Sang T, Zhao Q, Feng Q, Zhao Y, Li C, Zhu C, Lu T, Zhang Z, et al. Genome-wide association studies of 14 agronomic traits in rice landraces. Nat Genet. 2010;42:961-7.

35. Farneti B, Di Guardo M, Khomenko I, Cappellin L, Biasioli F, Velasco R, Costa F. Genome-wide association study unravels the genetic control of the apple volatilome and its interplay with fruit texture. J Exp Bot. 2017;68:1467-78.

36. Gabur I, Chawla HS, Snowdon RJ, Parkin IAP. Connecting genome structural variation with complex traits in crop plants. Theor Appl Genet. 2019;132:733-50. 
37. Mao H, Wang H, Liu S, Li Z, Yang X, Yan J, Li J, Tran LS, Qin F. A transposable element in a NAC gene is associated with drought tolerance in maize seedlings. Nat Commun. 2015;6:8326.

38. Liu S, Huang H, Yi X, Zhang Y, Yang Q, Zhang C, Fan C, Zhou Y. Dissection of genetic architecture for glucosinolate accumulations in leaves and seeds of Brassica napus by genome-wide association study. Plant Biotechnol J. 2019;18: 1472-84.

39. Zhou Z, Jiang Y, Wang Z, Gou Z, Lyu J, Li W, Yu Y, Shu L, Zhao Y, Ma Y, et al. Resequencing 302 wild and cultivated accessions identifies genes related to domestication and improvement in soybean. Nat Biotechnol. 2015;33:408-14.

40. Eduardo I, Pacheco I, Chietera G, Bassi D, Pozzi C, Vecchietti A, Rossini L. QTL analysis of fruit quality traits in two peach intraspecific populations and importance of maturity date pleiotropic effect. Tree Genet Genomes. 2010;7:323-35

41. Pirona R, Eduardo I, Pacheco I, Linge CDS, Miculan M, Verde I, Tartarini S, Dondini L, Pea G, Bassi G, et al. Fine mapping and identification of a candidate gene for a major locus controlling maturity date in peach. BMC Plant Biol. 2013;13(1):166.

42. Zhou H, Lin-Wang K, Wang H, Gu C, Dare AP, Espley RV, He H, Allan AC, Han Y. Molecular genetics of blood-fleshed peach reveals activation of anthocyanin biosynthesis by NAC transcription factors. Plant J. 2015;82:105-21.

43. Zhou H, Lin-Wang K, Wang F, Espley RV, Ren F, Zhao J, Ogutu C, He H, Jiang Q, Allan AC, Han Y. Activator-type R2R3-MYB genes induce a repressor-type R2R3-MYB gene to balance anthocyanin and proanthocyanidin accumulation. New Phytol. 2019;221:1919-34.

44. Tuan PA, Bai S, Yaegaki H, Tamura T, Hihara S, Moriguchi T, Oda K. The crucial role of PpMYB10.1 in anthocyanin accumulation in peach and relationships between its allelic type and skin color phenotype. BMC Plant Biol. 2015;15:280.

45. Lesley JW: A genetic study of saucer fruit shape and other characters in the peach. In Proc Am Soc Hortic Sci 1940, (Vol. 37, pp. 218-222).

46. Lopez-Girona E, Zhang Y, Eduardo I, Mora JRH, Alexiou KG, Arus P, Aranzana MJ. A deletion affecting an LRR-RLK gene co-segregates with the fruit flat shape trait in peach. Sci Rep. 2017;7:6714.

47. Wu S, Zhang B, Keyhaninejad N, Rodriguez GR, Kim HJ, Chakrabarti M, Illa-Berenguer E, Taitano NK, Gonzalo MJ, Diaz A, et al. A common genetic mechanism underlies morphological diversity in fruits and other plant organs. Nat Commun. 2018;9:4734.

48. Guo J, Cao K, Li Y, Yao JL, Deng C, Wang Q, Zhu G, Fang W, Chen C, Wang X, et al. Comparative transcriptome and microscopy analyses provide insights into flat shape formation in peach (Prunus persica). Front Plant Sci. 2017;8:2215.

49. Cao K, Li Y, Deng CH, Gardiner SE, Zhu G, Fang W, Chen C, Wang X, Wang L. Comparative population genomics identified genomic regions and candidate genes associated with fruit domestication traits in peach. Plant Biotechnol J. 2019;17:1954-70.

50. Murray MG, Thompson WF. Rapid isolation of high molecular weight plant DNA. Nucleic Acids Res. 1980;8(19):4321-6.

51. Wang L, Zhu G, Fang W, Cao K, Wang X, Chen C. Descripters and data standard for peach; 2005.

52. Verde I, Jenkins J, Dondini L, Micali S, Pagliarani G, Vendramin E, Paris R, Aramini V, Gazza L, Rossini L, et al. The Peach v2.0 release: high-resolution linkage mapping and deep resequencing improve chromosome-scale assembly and contiguity. BMC Genomics. 2017;18:225.

53. Li H, Durbin R. Fast and accurate short read alignment with burrows-wheeler transform. Bioinformatics. 2009;25:1754-60.

54. Chiang C, Layer RM, Faust GG, Lindberg MR, Rose DB, Garrison EP, Marth GT, Quinlan AR, Hall IM. SpeedSeq: ultra-fast personal genome analysis and interpretation. Nat Methods. 2015;12:966-8.

55. Jeffares DC, Jolly C, Hoti M, Speed D, Shaw L, Rallis C, Balloux F, Dessimoz C, Bahler J, Sedlazeck FJ. Transient structural variations have strong effects on quantitative traits and reproductive isolation in fission yeast. Nat Commun. 2017:8:14061.

56. Treepong P, Guyeux C, Meunier A, Couchoud C, Hocquet D, Valot B. panlSa: ab initio detection of insertion sequences in bacterial genomes from short read sequence data. Bioinformatics. 2018;34:3795-800.

57. DePristo MA, Banks E, Poplin R, Garimella KV, Maguire JR, Hartl C, Philippakis AA, del Angel G, Rivas MA, Hanna M, et al. A framework for variation discovery and genotyping using next-generation DNA sequencing data. Nat Genet. 2011;43:491-8.

58. Sedlazeck FJ, Rescheneder P, Smolka M, Fang H, Nattestad M, von Haeseler A, Schatz MC. Accurate detection of complex structural variations using single-molecule sequencing. Nat Methods. 2018;15:461-8.

59. Nguyen LT, Schmidt HA, von Haeseler A, Minh BQ. IQ-TREE: a fast and effective stochastic algorithm for estimating maximum-likelihood phylogenies. Mol Biol Evol. 2015;32:268-74.

60. Alexander DH, Novembre J, Lange K. Fast model-based estimation of ancestry in unrelated individuals. Genome Res. 2009;19:1655-64.

61. Price AL, Patterson NJ, Plenge RM, Weinblatt ME, Shadick NA, Reich D. Principal components analysis corrects for stratification in genome-wide association studies. Nat Genet. 2006;38:904-9.

62. Danecek P, Auton A, Abecasis G, Albers CA, Banks E, DePristo MA, Handsaker RE, Lunter G, Marth GT, Sherry ST, et al. The variant call format and VCFtools. Bioinformatics. 2011;27:2156-8.

63. Tian T, Liu Y, Yan H, You Q, Yi X, Du Z, Xu W, Su Z. agriGO v2.0: a GO analysis toolkit for the agricultural community, 2017 update. Nucleic Acids Res. 2017;45:W122-9.

64. Lipka AE, Tian F, Wang Q, Peiffer J, Li M, Bradbury PJ, Gore MA, Buckler ES, Zhang Z. GAPIT: genome association and prediction integrated tool. Bioinformatics. 2012;28:2397-9.

65. Koren S, Walenz BP, Berlin K, Miller JR, Bergman NH, Phillippy AM. Canu: scalable and accurate long-read assembly via adaptive k-mer weighting and repeat separation. Genome Res. 2017;27:722-36.

66. Kurtz S, Phillippy A, Delcher AL, Smoot M, Shumway M, Antonescu C, Salzberg SL. Versatile and open software for comparing large genomes. Genome Biol. 2004;5(2):R12.

67. Livak KJ, Schmittgen TD. Analysis of relative gene expression data using real-time quantitative PCR and the $2-\Delta \Delta C T$ method. Methods. 2001;25(4):402-8.

68. Sun HJ, Uchii S, Watanabe S, Ezura H. A highly efficient transformation protocol for Micro-Tom, a model cultivar for tomato functional genomics. Plant Cell Physiol. 2006;47:426-31.

69. Yao JL, Xu J, Cornille A, Tomes S, Karunairetnam S, Luo Z, Bassett H, Whitworth C, Rees-George J, Ranatunga C, et al. A microRNA allele that emerged prior to apple domestication may underlie fruit size evolution. Plant J. 2015;84:417-27.

70. Guo J, Cao K, Deng C, Li Y, Zhu G, Fang W, Chen C, Wang X, Wu J, Guan L, Wu S, Guo W, Yao J, Fei Z, Wang L. An integrated peach genome structural variation map uncovers genes associated with fruit traits. Sequence Read Archive: PRJNA630113. https://www.ncbi.nlm.nih.gov/bioproject/PRJNA630113. Accessed 29 July 2020. 
71. Guo J, Cao K, Deng C, Li Y, Zhu G, Fang W, Chen C, Wang X, Wu J, Guan L, Wu S, Guo W, Yao J, Fei Z, Wang L. An integrated peach genome structural variation map uncovers genes associated with fruit traits. Zenodo. https://zenodo. org/record/3953424. Accessed 29 July 2020.

72. Guo J, Cao K, Deng C, Li Y, Zhu G, Fang W, Chen C, Wang X, Wu J, Guan L, Wu S, Guo W, Yao J, Fei Z, Wang L. An integrated peach genome structural variation map uncovers genes associated with fruit traits. GitHub. https://github. com/ytguojian/Peach_SV. Accessed 29 July 2020.

\section{Publisher's Note}

Springer Nature remains neutral with regard to jurisdictional claims in published maps and institutional affiliations.

Ready to submit your research? Choose BMC and benefit from:

- fast, convenient online submission

- thorough peer review by experienced researchers in your field

- rapid publication on acceptance

- support for research data, including large and complex data types

- gold Open Access which fosters wider collaboration and increased citations

- maximum visibility for your research: over $100 \mathrm{M}$ website views per year

At $\mathrm{BMC}$, research is always in progress.

Learn more biomedcentral.com/submissions 